\title{
A Knowledge-Based Decision-Making Framework for the Design of Manufacturing Networks for Custom-Made Products
}

\author{
Dimitris Mourtzis and Michalis Doukas \\ Lab for Manufacturing Systems and Automation, University of Patras, Patras 26500, Greece
}

\begin{abstract}
Efficient design of manufacturing networks is paramount for a sustainable growth. The establishment of mass customization and the transition to personalization complicates design activities and leads to vast amounts of unexploited data. This research work aims to exploit existing knowledge for enhancing decision-making during the initial manufacturing networks design, which carry out custom orders of industrial equipment. A method developed into software is proposed, comprising a Genetic Algorithm with knowledgeenriched operators and an intelligent initialization algorithm that exploits existing planning knowledge. The validation of the method is performed using data from a high-precision mold-making manufacturer and its network of first-tier suppliers.
\end{abstract}

Keywords: Manufacturing Networks, Knowledge, Decision making.

\section{Introduction}

The establishment of mass customization and the gradual transition towards personalization affects manufacturing network design [1]. To achieve the desired variety, quick adaptation and re-configuration of production networks is necessary [2]. Also, vast amounts of planning-related data are generated daily, which however, remain unexploited. Reuse of past knowledge can improve decision-making, as design, planning, and operation activities of past and new projects, share numerous commonalities [3].

This research work presents a method for the initial design of manufacturing networks subject to unpredictable customer requirements for custom-made products. The suggested approach attempts to identify near optimum manufacturing network configurations for a specific custom product, while minimizing the total flowtime. It also allows the timely reconfiguration of the system to adhere to actual manufacturing capacity and availability. The method is validated with data from the mold-making industry.

\section{State of the Art}

Knowledge reuse in manufacturing has yet to reveal its full potential [5] after almost three decades of research [4]. Numerous approaches are available for knowledge reuse in product design [6], process planning [7] and part family representation [8]. 
Regarding the problem of design of manufacturing networks, the identification of optimum multi-stage, multi-product manufacturing network or system configurations, under hard pre- and post-condition constraints is strongly NP-Hard [9]. Enumerative methods are non-applicable for such problems; instead optimization methods are being applied, aiming at multi- or single-objective optimization. A GA was used in [10] for addressing to the job-shop scheduling problem. The method included random input turbulences, multiple criteria and multiple job routes. A scheduling approach was developed in [11] using a GA, to determine the efficient assignment of machines and workers in order to optimize performance criteria such as the mean flow time. The problem of scheduling of a Flexible Manufacturing System using a GA with knowledge based genetic operators is included in [12]. The way that these operators exploit past knowledge was unclear. The authors in [13] attempt to discover knowledge patterns that are generated in each generation of a GA using Rough Set Theory. The combined utilization of knowledge-based methods directly incorporated in the metaheuristic optimization algorithm is missing from the literature to the best of the authors' knowledge.

The presented research work combines knowledge-based techniques with an optimisation engine for supporting the initial design of manufacturing networks for custom-made industrial equipment. Existing knowledge regarding past design and planning projects is embedded in evolutionary operators in the form of rules. In addition, the formation of the initial GA population is pre-optimised, since similarity measurements against known planning cases are used. Based on successfully executed past planning projects, the search for optimum configurations for new problems becomes guided.

\section{Knowledge-Based Manufacturing Network Design}

The proposed method focuses on identifying near-optimum feasible selections of manufacturing nodes and suppliers, while minimising manufacturing flowtime for an engineer-to-order product. The structure of the manufacturing network is shown in Fig. 1.

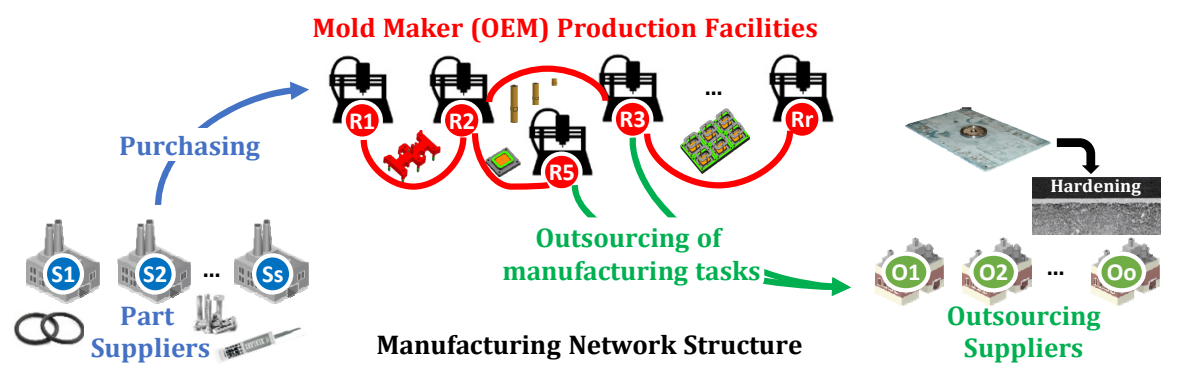

Fig. 1. The manufacturing network nodes

A number of processes are performed by the mold maker, while others, such as hardening of metallic components, are outsourced. Also, some components, such as guide columns, bolts, and sealant O-rings are purchased from $1^{\text {st }}$-tier suppliers. 
The facilities of the suppliers, as well as the individual machines possessed by the OEM, are characterised by an availability index that models manufacturing capacity to meet market demand. Static capacity models are widely used by industry as costeffective means for communicating capacity across the supply chain [17, 18]. The availability index values of OEM nodes and for suppliers are defined using historical observations.

\subsection{Formulation of the Design Problem}

Let the processing time for the task t to be performed by machine $\mathrm{k}$ be $\mathrm{p}_{\mathrm{tk}}$ and a task selection $p=\left\{p 1, p 2, \ldots, p_{n}\right\}$, where $n$ tasks $(j=1,2, \ldots, n)$ will be processed by $m$ machines $(\mathrm{k}=1,2, \ldots, \mathrm{m})$. Similarly, we define the setup times for machine $\mathrm{k}$ to perform task $\mathrm{t}$ as $\mathrm{s}_{\mathrm{tk}}$, and the waiting time of task $\mathrm{t}$ in the queue of machine $\mathrm{k}$ as $\mathrm{w}_{\mathrm{tk}}$. The sum of $\mathrm{w}_{\mathrm{tk}}, \mathrm{p}_{\mathrm{tk}}$ and $\mathrm{s}_{\mathrm{tk}}$ for all product components, represents the flowtime of the product. The Total Flowtime $\mathrm{FT}_{\text {tot(p) }}$ of a design $\mathrm{p}$ can be computed by summing all flow times (1):

$$
F T_{\text {tot }}=\sum_{t=1}^{\pi} F T\left(\pi_{t}\right)=\sum_{t=1}^{\pi} C\left(\pi_{t}, m\right)
$$

\subsection{Knowledge Enriched Genetic Algorithm}

The problem is solved by a Genetic Algorithm (GA) [14], with knowledge enriched operators. In contrast to a regular GA, the proposed enriched GA includes an intelligent initialization process and rule-based selection of genetic operators (selection, crossover and mutation). The steps of the knowledge enriched GA are visualized in Fig. 2.

Each manufacturing process can be performed by both a machine tool family and by specialized suppliers. However, based on technological differences and availability, different flow times are associated with different machines or suppliers carrying out the same task. Thus, each chromosome is encoded to represent alternative selections of nodes to carry out the required tasks. The encoding uses integer values in a matrix structure. This encoding structure is beneficial since it reduces decoding complexity.

\section{Initialization Procedure}

Instead of generating the initial population of alternative designs in a totally random fashion, each newly investigated planning case is compared to previously executed cases through a pairwise comparison of their most important attributes, i.e. number of components, special works, machining features, volumetric data, etc. Thus, based on similar past design cases, an appropriate adaptation of the task sequence and their mapping to processing nodes is done to best fit the new case requirements. This leads 
to a pre-optimised initial set of individuals to be fed to the GA. The similarity between numerical attributes is computed using the Euclidean distance (2).

$$
D_{n}=\sum_{i=1}^{n} \sqrt{|1-| 1-\frac{T_{p i}}{T_{n i}}\left|* w_{i}\right|}
$$

where: $\mathrm{n}=$ no. of features, $\mathrm{T}_{\mathrm{ni}}, \mathrm{T}_{\mathrm{pi}}=$ new and past case features, $\mathrm{w}_{\mathrm{i}}=$ feature weight.

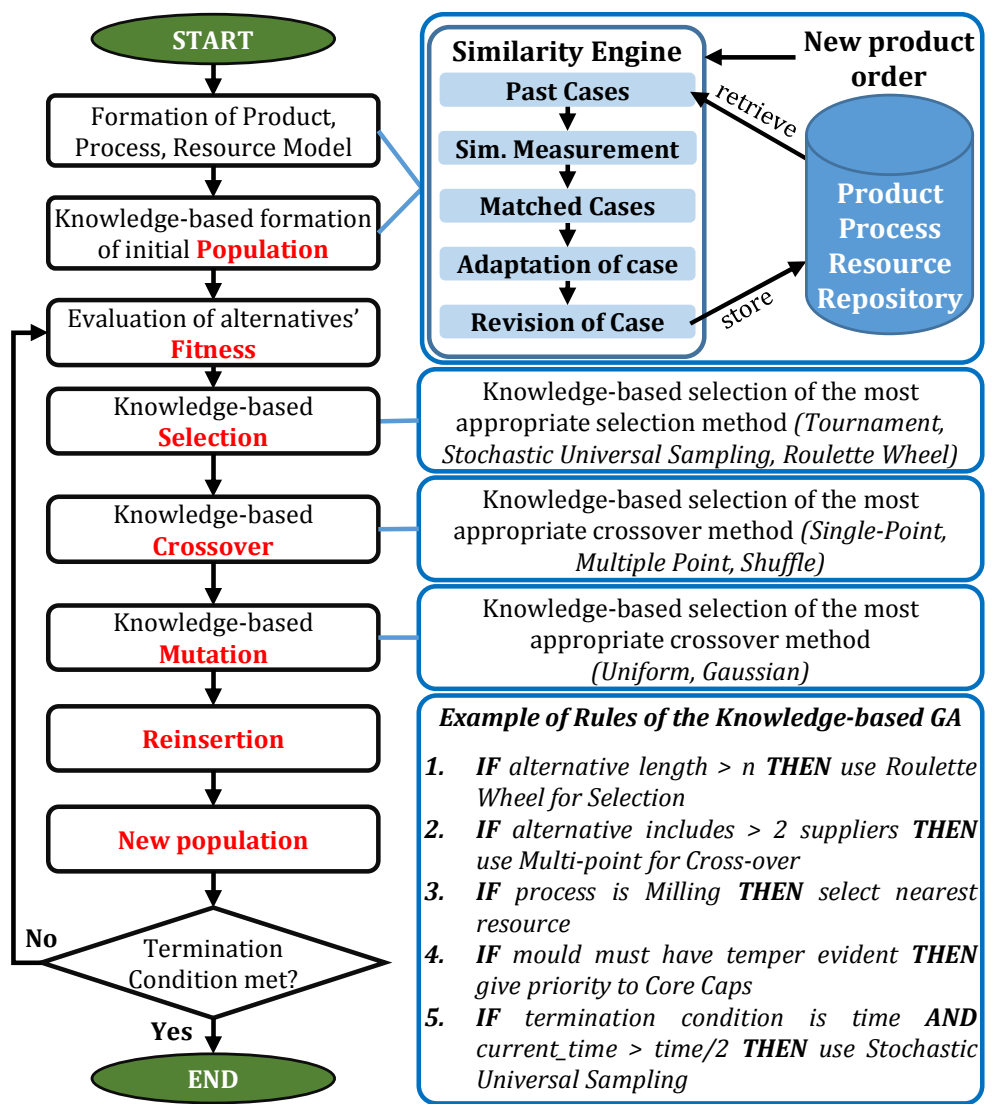

Fig. 2. The workflow of the knowledge enriched Genetic Algorithm

\section{Selection Operators}

Selection is used in GAs to identify individuals that will carry their genes to the next generation. Here, the following widely used operators Roulette Wheel Selection (RWS), Stochastic Universal Sampling (SUS) and Tournament Selection (TS) are used [15]. To exploit previous knowledge coming from network and human planner, and to imbue it in the algorithm, a set of IF THEN rules has been developed in cooperation with the industrial partner of the case study. An analysis involving semistructured questionnaires that were filled in by employees led to defining these rules. 
Moreover, literature findings about the performance of different operators in specific situations are encapsulated [15]. A rule example for adapting the selection method is the following:

IF current_CPU_time $<$ termination_CPU_time/5

THEN Selection_method $=R W S$

ELSE IF current_CPU_time > termination_CPU_time/2

THEN Selection_method $=$ SUS

ELSE Selection_method $=T S$

RLW is known to drive to convergence and SUS forces an even faster convergence by the algorithm. In case the predetermined runtime is near exhaustion, then SUS is assigned for deriving a solution. Additional rules are similarly implemented.

\section{Crossover Operators}

Crossover, handles mating between chromosomes, by selecting two (or more) parents in order to produce off-springs. The crossover operators used are: Single, Multi-point and Uniform. A rule example follows:

IF hardening_quality = very_good

\section{THEN Crossover_Method $=$ Single_Point_Crossover $(n)$}

Through a characterisation of hardening types (normal, good, very good) by experts, based on thickness quality (in $\mu \mathrm{m}$ ), specific partners must be selected that can offer the required precision. Due to the fact that these partners are located in distant countries from the mold maker facilities, hardening may require disproportionately large lead times (up to 3 weeks). Thus, a single crossover point is set right before the gene assignment that represents the partner that carries out the hardening. This ensures a higher degree of exploration of alternative paths and possibly great reductions in flowtime.

\section{Mutation Operators}

Mutation is necessary for maintaining the genetic diversity in the population. Fine tuning of mutation operators can alter the behavior of the search from explorative (visit new areas of the search space by creating new individuals) to exploitative (local optimization by fine-tuning existing individuals). The mutation operators are: Uniform and Gaussian Mutation. A rule for selecting each one of these methods is the following:

\section{IF Best_of_Pop $(n)<$ Best_of_Pop $(n-1)$ FOR 5 consecutive gens}

\section{THEN Mutation_Operator = Gaussian_Mutation}

In case the population stagnates to local optima and no improvement is observed in subsequent generations, then, a Gaussian mutation with reduced variance is selected, to ensure a higher probability of mutation compared to a purely stochastic sampler. 


\section{Industrial Pilot Case from the Mold Making Industry}

Mold making is technology, knowledge and labor intensive [16]. Each new mold is a unique product, which yet shares a number of commonalities with previous cases on process plan and resource level. Thus, reusing knowledge from finished mold-making cases can reduce overhead times required for initial network planning, decrease rampup, improve response time to volatile market requirements and lead to cost savings.

The case study for the validation of the method was an already finished mold with documented process plans from a company that manufactures high precision injection molds. The dataset included alternative machines and also operations and processing, setup and waiting times for similar machines to perform identical processes (Fig. 3).
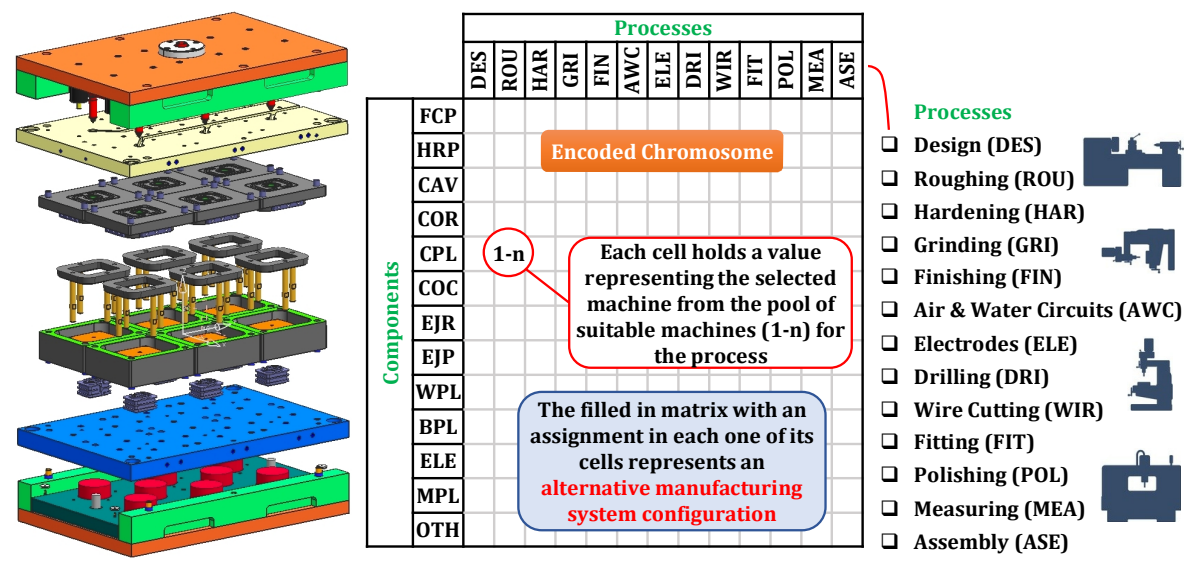

Fig. 3. The components of the mold and the integer encoding of the chromosome

The resource model of the pilot case includes the characteristics of the manufacturing nodes, and the required processing times of components in technologically similar resources, as well as the flowtimes required by suppliers for supplying the requested material or for performing a specialized process (e.g. hardening). Moreover, the availability of the resources and suppliers has been modelled based on historical data, as follows: $75 \%$ for part suppliers, 50\% for outsourcing suppliers, and 35\% for milling operations (roughing, finishing, air and water circuits cutting) performed in the manufacturing facilities of the OEM, $70 \%$ for wire cutting and die sinking, and $45 \%$ for manual tasks, such as fitting, polishing and assembly.

The product model includes: geometric characteristics of the components, number of cavities, tolerance specifications, cooling mechanisms, ejection methods and other process plan related information. The manufacturing processes required include: roughing, finishing, hardening, creation of air and water circuits, drilling, wire cutting, grinding, fitting, creation of electrodes for die sinking, polishing, measuring and assembly. 


\section{Experimentation: Results and Discussion}

A set of experiments were carried out to investigate the performance of the developed GA. The total number of feasible alternative configurations for this problem is $156 \times 10^{15}$, as a combinatorial analysis indicates. The optimal set of tuneable parameters of the GA was identified through a Statistical Design of Experiments (SDoE) and was the following: Population Size $=50$, No. of Generations $=50$, Generation Gap $=$ 0.9 , Termination Time $=20 \mathrm{sec}$ and Mutation Probability $=0.009$. Moreover, a correction factor is applied on the obtained flowtimes in order to take into consideration machine breakdowns, shortage of materials and other unpredictable factors. The correction factor was adjusted through the deviation of actual times obtained by the mold maker and experimental values for different mold cases. The results of the execution of the GA are shown in Fig. 4. The algorithm converges after 28 generations to an optimum flowtime of 1,211 hours requiring a total runtime of 19.56 seconds. In comparison, a regular GA (again with optimised parameters based on a SDoE) yielded in the same number of generations a globally best solution of 1,450 hours, i.e. a $17.96 \%$ worse solution. Most importantly, this obtained value is significantly lower $(38.26 \%)$ when compared to the actual flowtime for the specific order, which, as reported by the production engineer of the mold maker was 1,784 hours.

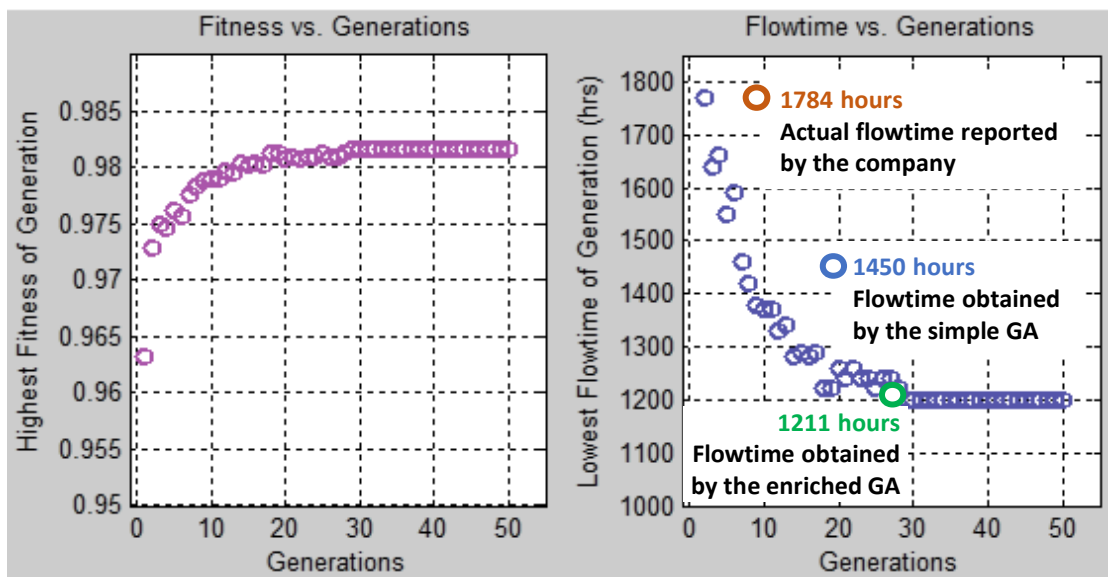

Fig. 4. Evolution of the fitness function (left) and improvement of total flowtime (FT) obtained by the enriched GA after 50 generations and comparison to historical data and a simple GA

\section{Conclusions and Future Work}

The presented research work investigated the initial design of a manufacturing network for custom-made products. The problem was tackled by a knowledge enriched Genetic Algorithm (GA) and its performance was tested in a real case study coming from the high precision mold making sector. The results depicted an improvement against both a regular GA and actual historical data. The encapsulation of knowledge 
into the genetic operators and the intelligent initialization process enhanced the robustness of the GA. The quality of the obtained solutions surpasses actual historical performance of the network, which was configured based on human experience and was therefore sub-optimal. Moreover, the small execution time of the algorithm facilitate the need for near real-time reconfiguration of the network for compensation to unforeseen disturbances. The proposed method and tool can be exploited by a planning manager in order to support the design and planning of efficient manufacturing networks and systems. The results obtained by the tool can be overridden by the manager in cases when the proposed solution is not satisfactory and the algorithm may be executed repeatedly until an accepted solution is found. The low computation requirements of the algorithm constitute it suitable for deployment on mobile devices and enables fast decisions on the go.

Future work will focus on extending the rule-base in order to cover additional historical observations and expert knowledge. Also, the method will be developed into an app for mobile devices. The GA will be executed into a company-owned private Cloud server and services will be exposed for distributing information to mobile devices.

Acknowledgements. This research work is partially supported by the EU funded research project "Applications for Advanced Manufacturing Engineering Apps4aME” (GA No. 314156).

\section{References}

1. Mourtzis, D., Doukas, M., Psarommatis, F.: Design and Operation of Manufacturing Networks for Mass Customisation. CIRP Annals 63(1), 467-470 (2013)

2. Mourtzis, D., Doukas, M., Psarommatis, F.: A multi-criteria evaluation of centralized and decentralized production networks in a highly customer-driven environment. CIRP Annals 61(1), 427-430 (2012)

3. Baxter, D., James, G.X., Keith, C., Jenny, H., Bob, Y., Sean, C., Shilpa, D.: An engineering design knowledge reuse methodology using process modelling. Research in Engineering Design 18(1), 37-48 (2007)

4. Chryssolouris, G., Wright, K.: Knowledge-based systems in manufacturing. Annals of the CIRP 35(2), 437-440 (1986)

5. Chryssolouris, G.: Manufacturing Systems: Theory and Practice, 2nd edn. Springer, New York (2006)

6. Cochrane, S., Young, R., Case, K., Harding, J., Gao, J., Dani, S., Baxter, D.: Knowledge reuse in manufacturability analysis. Robotics \& Computer-Integrated Manuf. 24(4), 508513 (2008)

7. Züst, R., Taiber, J., Schultschik, R.: Knowledge-Based Process Planning System for Prismatic Workpieces in a CAD/CAM-Environment. CIRP Annals 39(1), 493-496 (1990)

8. Mäntylä, M., Sohlenius, G.: Representation of Process Planning Knowledge for Part Families. CIRP Annals - Manufacturing Technology 42(1), 561-564 (1993)

9. Garey, M., Johnson, D.: Computers and Intractability - A Guide to the Theory of NPCompleteness, 1st edn. W.H. Freeman \& Co. Ltd., New York (1990) 
10. Chryssolouris, G., Subramaniam, V.: Dynamic scheduling of manufacturing job shops using genetic algorithms. J. of Intelligent Manufacturing 12(3), 281-293 (2001)

11. ElMaraghy, H., Patel, V., Ben Abdallah, I.: A Genetic Algorithm Based Approach for Scheduling of Dual-Resource Constrained Manufacturing Systems. CIRP Annals - Manufacturing Technology 48(1), 369-372 (1999)

12. Prakash, A., Chan, F.T.S., Deshmukh, S.G.: FMS scheduling with knowledge based genetic algorithm approach. Expert Systems with Applications 38(4), 3161-3171 (2011)

13. Yan, G., Xie, G., Chen, Z., Xie, K.: Knowledge-Based Genetic Algorithms. In: Wang, G., Li, T., Grzymala-Busse, J.W., Miao, D., Skowron, A., Yao, Y. (eds.) RSKT 2008. LNCS (LNAI), vol. 5009, pp. 148-155. Springer, Heidelberg (2008)

14. Holland, J.: Adaptation in Natural and Artificial Systems. University of Michigan Press (1975)

15. Mitchell, M.: An Introduction to Genetic Algorithms. The MIT Press, Cambridge (1999)

16. Klocke, F., Bilsing, A., Wagner, C.: Perspectives for the German Die and Mold Manufacturing Industry. Werkstatt und Betrieb 133(5), 18-21 (2000)

17. Witte, J.D.: Using static capacity modeling techniques in semiconductor manufacturing. In: Proceedings of the IEEE/SEMI 1996 Advanced Semiconductor Manufacturing Conference and Workshop, ASMC 1996, pp. 31-35. IEEE (1996)

18. Ozturk, O., Coburn, M.B., Kitterman, S.: Conceptualization, design and implementation of a static capacity model. In: Proceedings of the 2003 Winter, vol. 2, pp. 1373-1376. IEEE (2003) 